\title{
Research on application of ground penetrating radar method in detecting side walls and small targets of Karst tunnel
}

\author{
Shanshan Gao , Wei Li, and Wenhua Zhu \\ SINOHYDRO BUREAU 14 Co., LTD., Kunming, Yunnan, 650041, China
}

\begin{abstract}
Keywords: karst, ground penetrating radar detection, side walls detection, small targets detection.

Abstract. In the tunnel projects construction process of our country, there are many crossing karst areas situations. Advanced detection of abnormal geological bodies in karst areas is essential to ensure the safety of tunnel construction. This paper takes Guizhou Provonce Expressway Project-Xinjie Tunnel as an example. By studying the overall karst situation of Xinjie Tunnel, the basic principles of Ground Penetrating Radar detection and tunnel detection principles are explained. The detection methods and effects of the Side Walls and Small Targets are explained by real examples. Advanced detection of the abnormal geological bodies that may be encountered in the tunnel construction and trying to avoid the possible geological disasters in the tunnel construction process, are very important to provide guarantee for the safety of tunnel construction.
\end{abstract}

\section{Introduction}

The Xinjie Tunnel is located in Fuquan City, Guizhou Province, China and is a separate super long tunnel.The length of the tunnel is $3403 \mathrm{~m}$.

The main section of the tunnel is dominated by limestone, including a small amount of mudstone, and karst is developed throughout the section. According to the comprehensive research results, the entire section of the Xinjie Tunnel passes through the soluble rock area. The site karst is extremely developed, and the caves and pipelines are interconnected, forming a developed underground cave and pipeline network. The possibility of exposing hidden karsts (karst caves, karst cracks and karst pipelines, etc.) during construction is extremely high, and engineering geological disasters such as collapse, sudden water inrush, sand inrush, mud outburst and even roof fall are easily to occur. Due to the extremely strong development of karst in the site, under the action of heavy rainfall in the rainy season, surface water will quickly be converted to groundwater through various channels mainly karst pipelines. If the karst pipelines are exposed during tunnel construction, the risk of mud and water inrush will be greatly increased.

\footnotetext{
*Corresponding author: 18010016358@163.com
} 


\section{Basic principles of ground penetrating radar detection}

Ground Penetrating Radar (GRP) is an electromagnetic wave detection technology that detects the electrical differences of different underground media. It has the advantages of non-destructive detection, fast detection speed, high resolution and sensitivity to water bodies. It has been extensively studied and applied in tunnel construction safety monitoring and advanced detection of abnormal geological bodies. The Ground Penetrating Radar uses an antenna to transmit electromagnetic waves with the same center frequency to the target formation at a certain frequency. When the electromagnetic waves travel in the target formation, if there are geological abnormalities in the target formation, the electromagnetic wave properties of the target formation will change, it makes electromagnetic waves to be reflected and refracted at the interface of abnormal geological bodies, and the reflected waves will be received along the echo path. By analyzing the waveform characteristics of the reflected wave, the approximate development of abnormal geological bodies in the target formation can be detected. (The basic working principles and compositions of the Ground Penetrating Radar are shown in Figure 1).

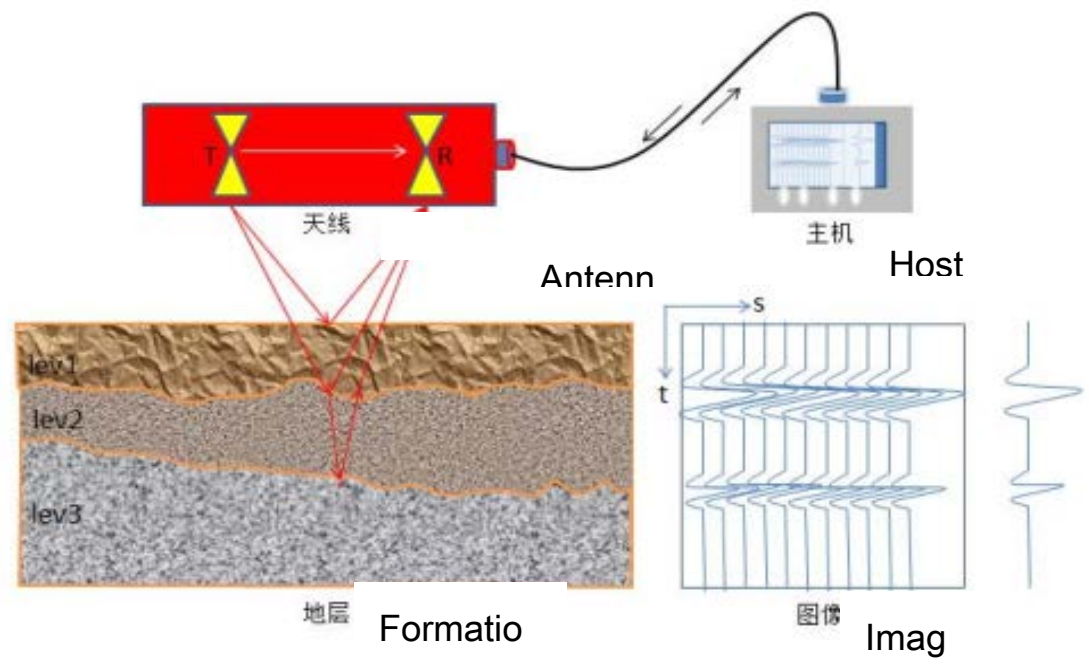

Fig. 1. The basic working principles and compositions of the Ground Penetrating Radar.

During the detection process of Ground Penetrating Radar, the reflection characteristics of reflected waves are the keys to the detection of underground targets. In most cases the electrical parameters are directly related to the reflection characteristics. The main electrical parameters include three: dielectric constant, electrical conductivity, and magnetic conductivity.

\section{Tunnel ground penetrating radar detection principles}

Electromagnetic waves are susceptible to be disturbed from metal objects, noise and other interference sources during the traveling process. According to existing research, in order to ensure and improve the accuracy of on-site detection, combined with the specific application effects in the Xinjie tunnel, it is believed that the following principles should be noted.

(a) Interference Sources Avoiding Principles

The Ground Penetrating Radar is susceptible to the influence of trolleys, bolts, drill pipes and other metal construction tools near the tunnel face during detection. The specific 
manifestation mode is that the reflected signal of interference sources will appear on the radar reflection profile. If you do not pay attention to the The identification and elimination of the interference source signal can easily misjudge the interference signal as the reflected signal of the surrounding rock, which may mislead the interpretation.

On the other hand, the Ground Penetrating Radar's signal system uses a wide-band recording mode. So when there are noise sources around the tunnel face, such as noise, mobile phone signals, car engines, etc., if the frequency band of these interference sources is just within the geological radar's recording frequency range Inside, these noise signals will also be recorded in the radar reflection profile. Therefore, in actual detection by the geological radar, pay attention to clear the surrounding interference sources in time is very necessary.

(b) Applicability principles

The absorption and attenuation of electromagnetic waves are decided that the electromagnetic waves whether can continue to effectively travel in front of the palm face. First, the electromagnetic wave is emitted by the radar antenna, then passes through the air to the palm face, and finally enters the surrounding rock through the palm face.

Engineering practice shows that when there is water gushing, water seepage or diffuse water on the surface of the palm face, or there is no obvious water seepage but the surface of the palm face is obviously wet, or the surface of the palm face contains a plastic to flow-plastic clay covering layer, the tunnel palm face will strongly absorb electromagnetic waves, which will cause the electromagnetic waves decay rapidly. Therefore, in actual detection of the tunnel palm face, special attention should be paid to the water content of the tunnel palm face. When the tunnel palm face is in a high water content state, the Ground Penetrating Radar detection is likely to fail.

\section{(c) Ambiguity Principles}

The accuracy of the Ground Penetrating Radar detection result is related to multiple factors such as whether the detection method is correct and whether it is interfered by external interference sources. Any mistakes may lead to the ambiguities of the Ground Penetrating Radar interpretation. Therefore, the Ground Penetrating Radar detection method requires high engineering experience and professional level of the detection personnel. In actual detection, professional operators with rich engineering experience and high theoretical level should be selected to carry out the detection work.

In the actual field detection of Ground Penetrating Radar, In order to improve the accuracy of field detection, we should pay attention to the above principles.

\section{Special geological bodies at close range of side wall position detection}

During the construction process of the tunnel, the scale of the abnormal geological bodies are complex and changeable, and the distribution location also varies. According to the propagation characteristics of electromagnetic waves that any single kind of survey line or detection parameters are generally difficult to distinguish targets of different positions or sizes.

When the surveying points are arranged in the tunnel palm face, due to the limitation of electromagnetic attenuation and "footprint", compared with the abnormal geological bodies within the tunnel palm face, the abnormal geological bodies at the side wall position are often difficult to be identified. According to the footprint limitation theory, according to the axial distance between the abnormal geological body and the palm face of the tunnel, the abnormal geological body within $5 \mathrm{~m}$ is called the abnormal geological body close to the side wall position, and the theoretical analysis and actual measurement are applied to this research and analysis of on-site detection methods for similar abnormal geological bodies. 
For the problem that the Ground Penetrating Radar is difficult to detect the abnormal geological body of the side wall within $5 \mathrm{~m}$ in front of the tunnel palm face, based on the theoretical analysis and practical detection, the research and analysis have obtained a more effective detection method, and the detection has been well applied in the advanced abnormal geological bodies detection of the Xinjie tunnel, and the overall detection rate of abnormal geological bodies in the side wall is nearly $80 \%$. For example, Both in the YK48+080 mileage at the right entrance of the Xinjie Tunnel and the ZK50+837 mileage at the left exit of the Xinjie Tunnel Karst cave in the side wall within $5 \mathrm{~m}$ in front of the palm face were mostly detected.

Figure 2(a) shows the cave detected at the YK48+080 mileage on the right side of the Xinjie Tunnel entrance. The cave is about 1.5 meters wide and 4 meters high. It is filled with clay and a large amount of calcite is developed in the surrounding rock on the left side of the cave. The waveform diagram is shown in Figure 3(a). The advanced prediction results are: the surrounding rock in the prediction section is moderately weathered limestone, with mud interspersed, the rock is harder, the joints and fissures are much more developed, the rock mass is easy to broken, and the surrounding rock stable ability is Poor. YK48+080 YK48+083 mileage have developed dissolution fissures and may develop dissolution caves.

Figure 2(b) shows a vertical cave detected at the ZK50+837 mileage on the left panel of the Xinjie Tunnel exit. The cave is located near the wall on the left side of the tunnel palm face, with a diameter of about $1 \mathrm{~m}$, and the inside space of the cave is filled with yellow fully weathered mud. The waveform diagram is shown in Figure 3(b). The advanced prediction results are: the surrounding rock in the forecast section of ZK50+837 ZK50+807 mileage on the left is intense to moderately weathered limestone, with mud interspersed, high shale content, and rock quality It is relatively hard, with well-developed joints and fissures, broken rock mass, and poor stability of surrounding rock. The rock masses in the detection section $2 \sim 10 \mathrm{~m}$ are broken, dissolution fissures are developed, and karst caves are developed, filled with mud; 14 27m dissolution fissures are developed, and small cavities may develop locally.

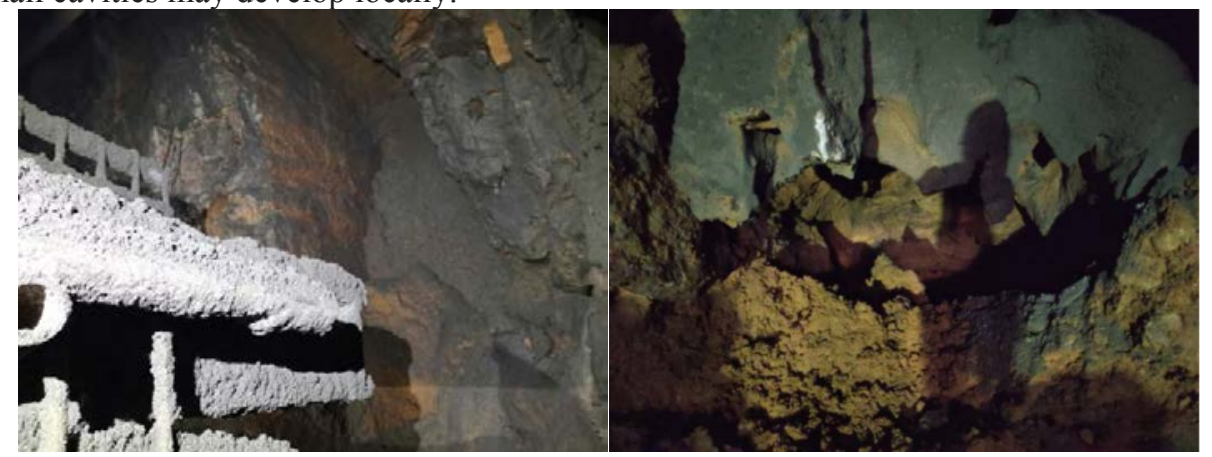

(a) Karst caves on the right wall of the entrance of Xinjie Tunnel YK48+080 mileage (b) Karst cave on the left side of Xinjie Tunnel ZK50+837 mileage

Fig. 2. Examples of abnormal geological close-up of the side wall in front of the palm face. 


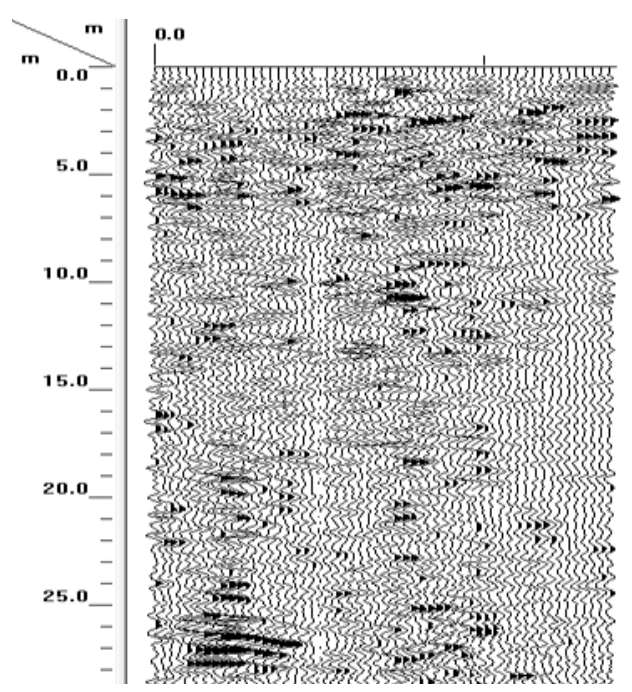

(a) Xinjie Tunnel YK48+080 YK48+110

mileage detection waveform

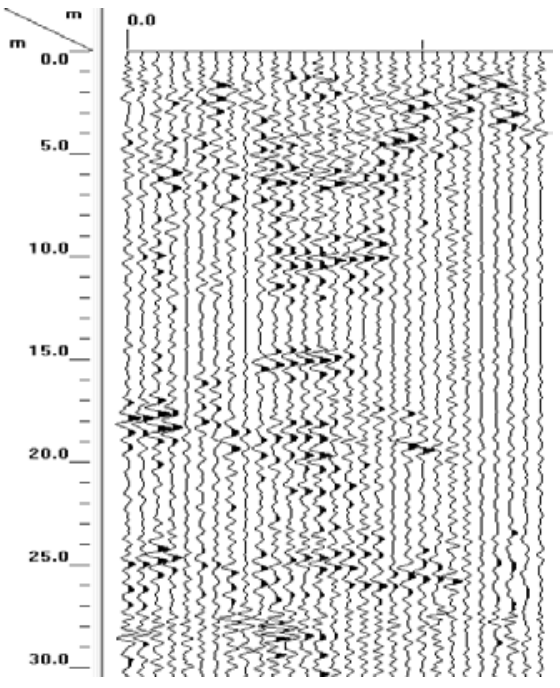

(b) Xinjie Tunnel ZK50+837 ZK50+807

mileage detection waveform

Fig. 3. Xinjie Tunnel Detection Waveform.

\section{Small target detection within the palm face}

For small targets within the palm face, the survey line measurement points can generally be arranged in most places of the palm face. So, compared with the abnormal geological body within the side wall, when detecting small targets within the palm face The detection key is to meet the detection depth and resolution.

According to existing research and actually detection, when the palm face is relatively complete and dry, the $100 \mathrm{MHz}$ antenna can identify the target with a vertical diameter of less than $30 \mathrm{~cm}$.

This detection scheme has been well applied in the construction of Xinjie Tunnel, and a large number of small targets have been detected and identified, especially small caves, small fracture zones and fracture zones.

Figure 4(a) shows that at the YK49+580 mileage on the right side of the Xinjie Tunnel exit, a mud-filled cave with a width of about 2 meters is detected in the middle of the tunnel palm face, and a vertical mud-filled fissure is developed on the left, with a width about $10 \mathrm{~cm}$. The waveform diagram is shown in Figure 5(a). The advanced prediction results are: It is estimated that the surrounding rock in the detection section of YK49+580 $\sim$ YK49+550 mileage on the right of the exit is moderately weathered limestone with relatively hard quality. The radar waveform is disordered, the event axis is broken, and strong reflections can be seen partial place. The reflections at $3 \sim 6 \mathrm{~m}$ and $16 \sim 18 \mathrm{~m}$ place are enhanced. so, it is inferred that the dissolution fissures are developed at YK49+577 YK49+574 and YK49+564 YK49+562, and filled with mud. Joint fissures are also developed and filled with mud, the rock mass is broken, the surrounding rock has poor self-stabilization ability, and the groundwater is relatively developed.

Figure 4(b) shows a developed karst cave detected at the left side of the ZK48+389 mileage at the entrance of the Xinjie Tunnel, filled with mud, extending $45^{\circ}$ diagonally upward to the right, about $11 \mathrm{~m}$ high and about $7 \mathrm{~m}$ deep, and the width is about $5 \mathrm{~m}$. The waveform diagram is shown in Figure 5(b). The advanced prediction results are: the surrounding rock in the forecast section is medium to slightly weathered limestone, with 
partial mud interspersed, the rock quality is relatively hard, the joints and fissures are more developed, the rock mass is relatively broken, and the surrounding rock Poor self-stabilization ability. The prediction section $8 \sim 14 \mathrm{~m}, 18 \sim 24 \mathrm{~m}$ develops dissolution fissures which filled with mud, and it is possible to develop a clay-filled solution cave. The groundwater is not developed.

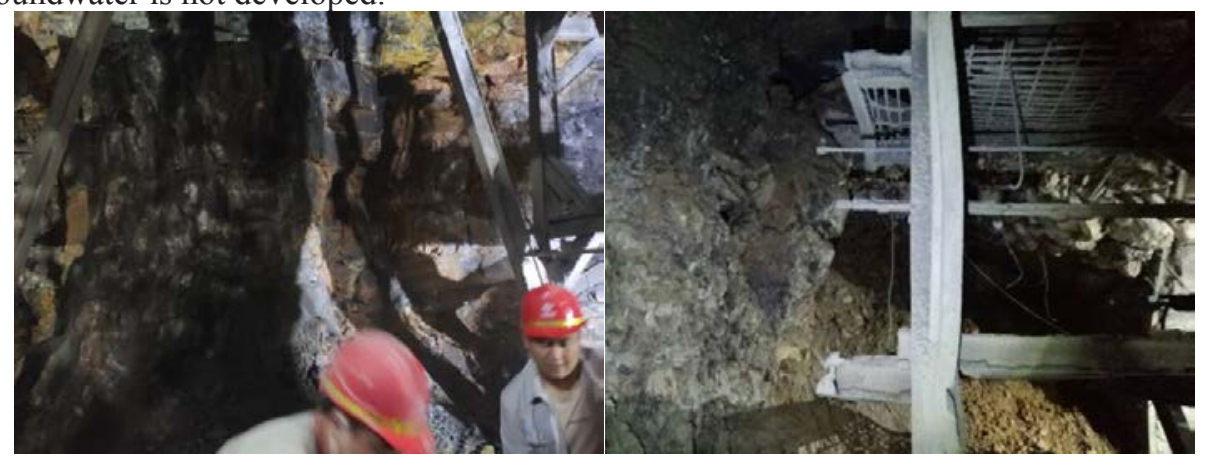

(a) Xinjie Tunnel YK49+580 mileage central karst cave (b) The karst cave on the right side of the tunnel face ZK48+389 mileage of Xinjie Tunnel

Fig. 4. Some small targets actually detected.

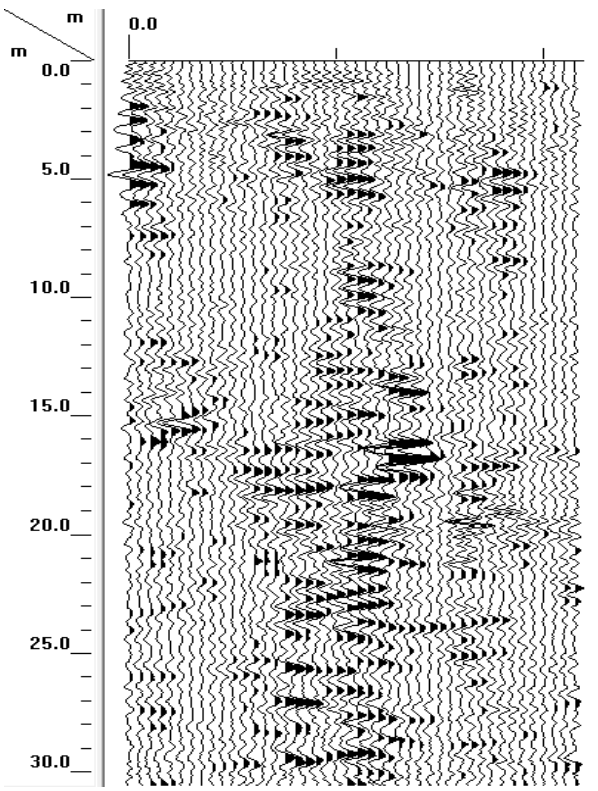

(a) Xinjie tunnel YK49+580 YK49+550 detection waveform

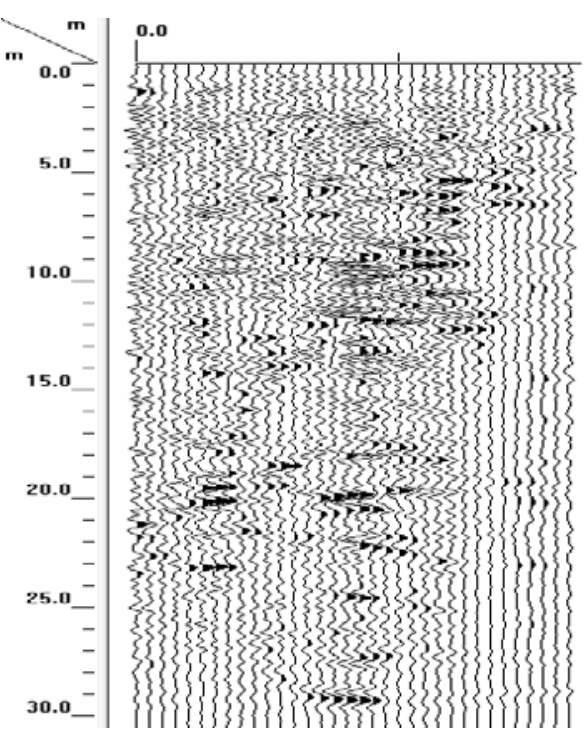

(b) Xinjie tunnel ZK48+389 ZK48+419 detection waveform

Fig. 5. Xinjie Tunnel Detection Waveform.

Due to the small size of small caves and other small targets, the radar antenna is limited by resolution and detection depth. It is difficult to meet the requirements for detection depth and resolution at the same time by using a single antenna. Using antennas combined with different center frequencies to detect scheme can solve the problems of detection depth and resolution at the same time. The successful examples in the Xinjie Tunnel show the feasibility and effectiveness of this project 


\section{Summary}

Karst develops in the whole section of Xinjie Tunnel. Through the judgment and analysis of the surrounding rock and Ground Penetrating Radar waveform of the tunnel palm face, and combined with the actual construction situation on site, it can be found that using the Ground Penetrating Radar detection method to identify karst tunnel side walls and small targets has a good effect. Although the detection of side walls is limited by location factors which make it not easy to detect, from an overall point of view, the Ground Penetrating Radar method has a better detection results and a higher accuracy rate, which can achieve the purpose of guiding construction. So it is an effective and safe prediction method in karst tunnel construction.

\section{References}

1. Forecast of Karst-Fractured Groundwater and Defective Geological Conditions [J]. Li Shucai, Li Shuchen, Zhang Qingsong, Xue Yiguo, Ding Wantao, Zhong Shihang, He Faliang, Lin Yushan. Chinese Journal of Rock Mechanics and Engineering. 2007(02).

2. Comprehensive Advanced Geological Prediction Method for Super Long Tunnel and it's Application[D]. Liu Yangfei.Chengdu University of Technology. 2016.

3. Study on Application of Advanced Construction Geological Prediction for an Expressway Tunnel[D]. Wang Ning. South China University of Technology. 2014.

4. Application of Geological Prediction to Tunnel Engineering Construction[J]. Wu Jiangbin. Journal of Engineering Geology. 2003(02).

5. Comparison of Techniques for Geological Prediction of Tunnels [J]. Chen Jianfeng. Underground space.2003(01).

6. Research and Application of Geological Radar in Advance Detection of Highway Tunnel in Karst Area[J]. Wu Zhenwei, Pan Longwu, Deng Jiaxi. Western Transportation Technology. 2017(06)

7. Hydrologic and Geological Forecast of Tunnel Construction in the Karst District [J]. Wang Mengshu. Railway survey. 2004(01).

8. Integrated advanced geological prediction technology of tunnel and its engineering application[J]. Zhou Lun, Li Shucai, Xu Zhenhao, Li Liping, Huang Xin, He Shujiang, Li Guohao. Journal of Shandong University (Engineering Science Edition). 2017(02).

9. Application research of geoligy forecast by Ground Penetrating Radar on karst tunnel $[\mathrm{J}]$. Jiang Hongliang, Yang Tingwei, Lu Chaobo. Engineering Technology Research. 2018(11).

10. Application of geological prediction in tunnel construction[J]. Bao Tai. Journal of Pingdingshan Institute of Technology. 2008(01)

11. Application of Ground-penetrating Radar in the Geological Prediction of Tunnel in Karst Area[J].Su Tao, Hou Junmin, Che Ligang. Highway Engineering.2018(04). 